\title{
Simulation of the technological process for stone processing
}

\author{
Nikoleta Mikušováa ${ }^{1,}$, Michal Hozza ${ }^{1}$, and Roland Salai ${ }^{1}$ \\ ${ }^{1}$ TU Kosice, Faculty of Mining, Ecology, Process Control and Geotechnologies, Letná 9, 04201 Košice, \\ Slovakia
}

\begin{abstract}
Simulation and modelling is an important part of all technicians and engineers working in different industries. For these tasks, it is important to have the necessary data and their application in programs and tools. The paper deals with simulation of the production process of stone in the selected enterprise in Slovakia. The simulation model can realize an experiment without intervention to the operation. The study analyses the production process of stone processing, from primary to secondary stone processing. The result of this study is presented by simulation experiments of production processes.
\end{abstract}

\section{Introduction}

Modelling and simulation as scientific methods are expanded in the area of science and research. One of the basic contributions of modelling and simulation is presented by material and energy savings. The contribution is also a more efficient production or distribution in practice. It is possible to monitor situations when the original amount of machines does not process all supplies for customers. The material that is intended for processing, must wait. For that reason, analysis and simulation of the production process are very important for prevention of ineffective using of machines and increasing of the efficiency of the stone processing process [1].

Simulation is a representation of a real system by a simulation model describing the properties of the real system. The simulation experiment suggests various improvements of the simulated system and verifies the impact on the modelled system. The results are backwards applied to the real system and improve its properties. The simulation does not allow to get an optimal solution but allows to test effects of decisions on the simulation model. The results obtained from the simulation are probably the values of the simulation model and it is necessary to work with them in the next use. The simulation model can but does not need and usually does not start from the mathematical description of the simulated system. One of the important properties of the simulation model is simulating of a simulated system with the same organizing of changes in the time [2]. The simulation allows experimenting outside a real object. The term simulation is possible to explain using synonyms as to pretend and imitate. The simulation is the method by which the dynamics

\footnotetext{
* Corresponding author: nikoleta.mikusova@,tuke.sk
} 
system is replaced by its simulator and experiments are realized with them and the aim is to obtain information about the original examined system. It is possible to characterize a simulation with three steps: 1. Formation of a simulation model of a real system, 2 . Realization of experiments with a simulation model, 3. Backward use of the obtained results for improving the system. The process of creating of the simulation model is presented by 1 . Definition and formalization of the problem, 2. Analysis of the problem, 3. Formalization of the model, 4. Parameters estimation and limitation of the model, 5. Algorithm and flow diagram construction, 6 . Transcription of the algorithm to the program, 7. Realization of the simulation calculation, 8. Verification and analysis of the results [3]. A computer simulation in certain cases helps to realize a general scientific method such as analysis. The analysis helps to reveal more complex dependencies, and also to investigate the dynamics of the system for different cases. The deductive deduction and use of strict deterministic models are not useful by the system with random variables. A simulation is a process of designing a real system and realization of experiments with this model in order to understand system behaviour, evaluation of different strategies for system operation. A simulation of a system can be realized without real operation and the existence of a real system. Information about the examined objects of the system can be obtained by experimenting with a real system, physical model, real system, experimenting with the model on the base of simulation, experimenting with the model created from the mathematical model and its solution with available methods. The simulation can be defined as the method of processes investigation, in order to obtain models in the examined process. Strengths of the computer simulation are presented by these facts: it allows to solve analytically insolvable tasks, it makes easier the solution of hardly solvable tasks, it standardizes some qualitative parameters, the process of model creation helps to discover and specify new facts. Weaknesses of the computer simulation are presented by these facts: disadvantages resulting from general well-known problems of the model using, the possibility of failure in the process of simulation of the extreme events, the possibility of subjective manipulation with the model, methodological fastidiousness of the method, the method of computer simulation is not general [4].

\section{Stone processing in the examined enterprise}

The examined enterprise uses two ways of processing and production of the stone: primary stone production and secondary stone production. Primary stone production consists of three processes: 1. extraction and loading of the stone block in the quarry, 2. transport of the stone block to the primary stone production for the next processing, 3 . The process of semi-finished products from the stone blocks. The block of stone is processed by the technique: a. pressing mould to the tables with the thickness 20, 30, 40, 50, $60 \mathrm{~mm}$ (Fig. 1), b. circular large-average saw with the diameter of the circular $\Phi 3000$ (Fig. 1), 2500, 2000, $\mathrm{mm}$ to the tables with the thickness $100,200,300 \mathrm{~mm}$, c. multi-circular saw with the diameter of the circular $\Phi 900 \mathrm{~mm}$ to the belt with the width $300 \mathrm{~mm}$, thickness 10 and $12 \mathrm{~mm}$. The secondary stone production consists of these steps: 1 . manipulation of semi-finished products by the final producer, 2 . process of premium stone production.

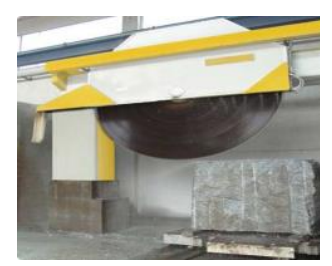

Fig. 1. Circular large-average saw (diameter of the circular $\Phi$ 3000). Source: Authors 
The material (stone) can be processed by cutting into tables or belts. The surface can be processed in two ways: 1. polishing - it is the basic and the most common method of the surface treatment for natural stone. Gradual polishing results in a mirror surface gloss. Polished surface highlights the nature of the natural stone. The block of the stone for production is divided into two categories: polishing belts (Fig. 2) and polishing tables (Fig. 3). The block of the stone for processing, polishing belts are made by multi-plate cutting machine to dimensions that are normalized. The block of stone is processed by a stone cutting machine, the results are polished tables with standardized sizes.

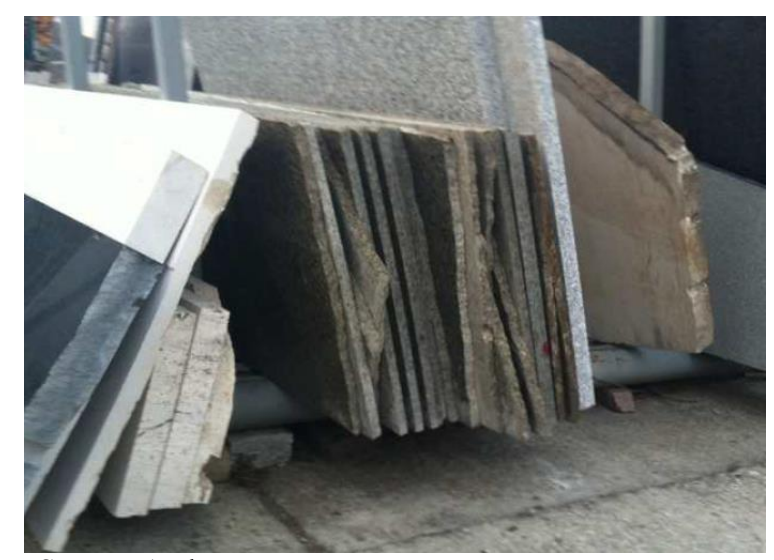

Fig. 2 Polishing belts. Source: Author

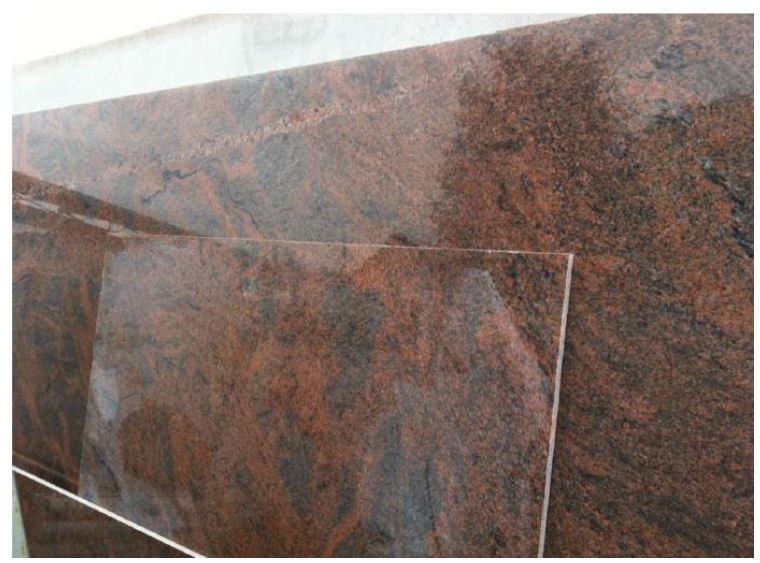

Fig. 3 Polishing tables. Source: Authors

2. Flaming - it is a process in which the stone (granite) is treated with high heat. The surface of the stone becomes rough. Flaming is also the most common form of creation of anti-skid surface for natural stone. The surface after this process is rough and anti-skid. The block of the stone for production is divided into two categories: flaming belts (Fig. 4) and flaming tables (Fig. 5). Flaming belts are made by multi-plate cutting machine and tables are made by stone cutting machine. 


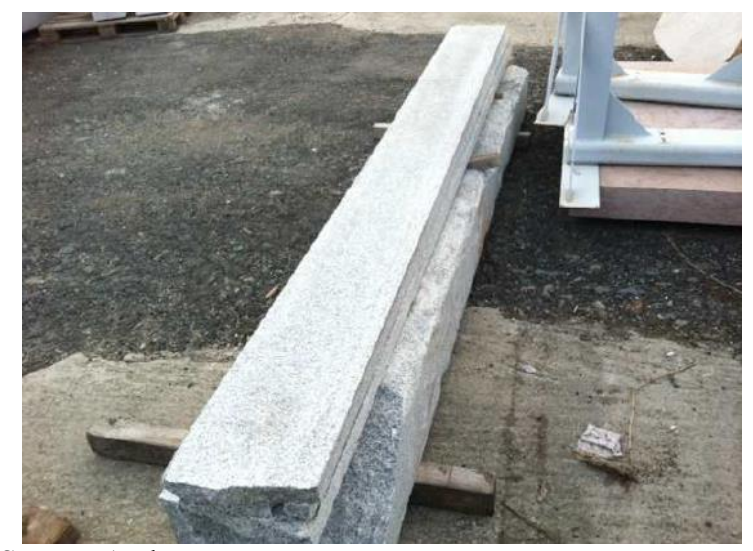

Fig. 4 Flaming belt. Source: Authors

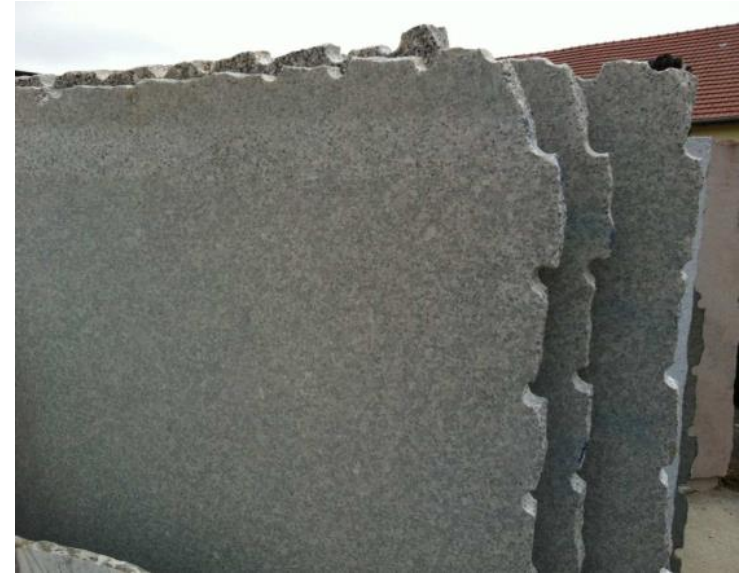

Fig. 5. Flaming table. Source: Authors

\section{A simulation model of the process of stone processing in the selected enterprise}

The simulation language EXTEND was used to analyse the process of stone processing. This model (Fig. 6) uses discrete and continuous blocks and the model consists of these blocks: 1. Block "Activity Delay" - this block suppresses some requests during the defined time. The block uses a simulation of the machine in the model and allows to set the time of processing of the request on the machine. The time of processing can be set in the folder activity; 2. Block "Combine" - it allows to connect two input requests from two different sources to the one input flow; 3. Block "Executive" - it forms the base of each discrete model. This block sets the time to end the simulation; 4. Block "Generator" - it completes the function of the generator of input request to the system by the defined distribution of the random variable; 5. Block "Set Attribute" and "Input Random Number" - the block Set Attribute" assorts the attribute to the request. The block "Input Random Number" sets percentage representation of the material with the appropriate value; 6. Block "Queue FIFO" - this block has the function of the request restraint. It is possible to set the needed number of requests which are waiting for the processing in this block; 7. Block "Get Attribute" - it is a reader of the set attributes of the previous requests; 8. Block "Throw" and "Catch" before setting of the block "Throw" it is necessary to name all branches for transfer of request 
of the block "Catch". Every part of the block "Catch" must have an equal name as it was defined in the block "Throw"; 9. Block "Count Items" - this block is a service for displaying of scaled values of the requests; 10. Block "Exit" - it has a function of removal of all requests (input requests) and it also processes the statistics of the processed requests; 11. Block "Decision" - this block compares values in the input A and B by the defined condition; 12. Block "Equation" - it allows calculating the input value on the base of the defined equation. The equation can use values from five inputs; 13. Block "Holding Tank" - this block allows to set the initial value; 14. Block "Meter" - this block can help to set max. and min. value of displaying; 15. Block "Plotter" - the block helps to graphically display the simulation.

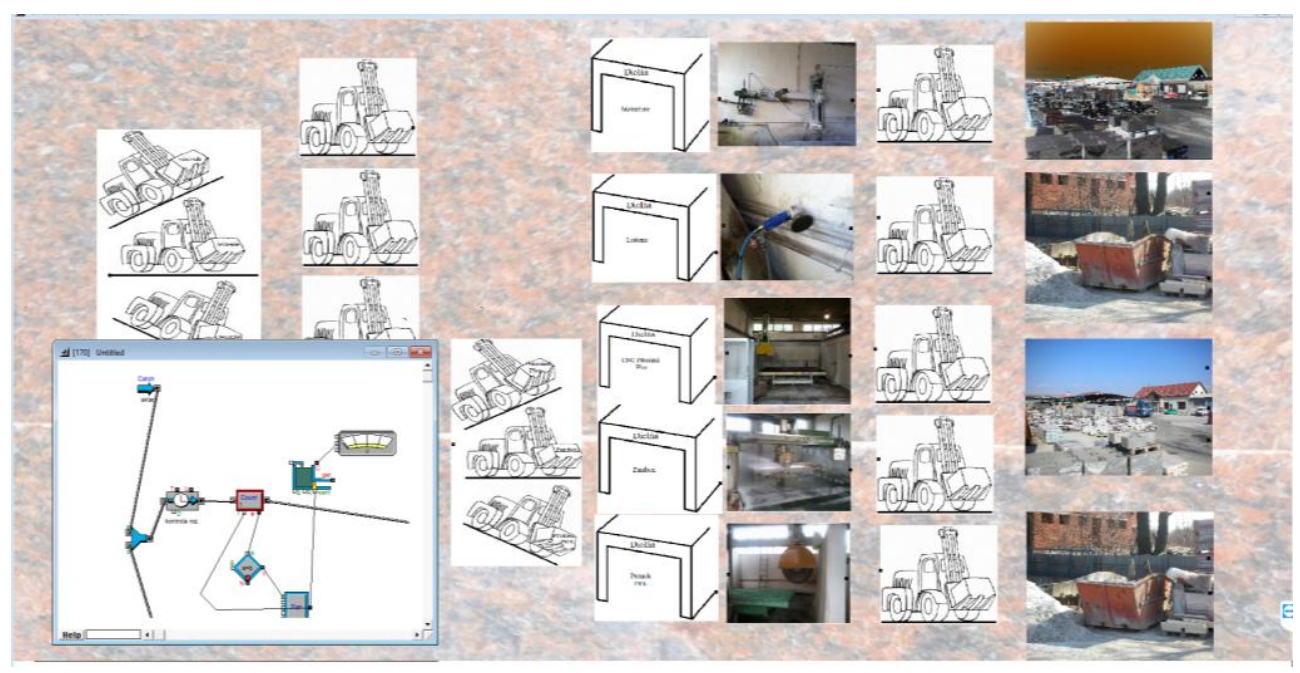

Fig. 6. Simulation model. Source: Authors

\subsection{Simulation experiment}

The simulation language EXTEND was used for analysis of the production process (its primary and secondary part). This simulation language helps to process a large quantity of material per time unit one year in a relatively short time. The obtained information about the processed amount of material in $\mathrm{m} 2$ from the simulation was compared with the real values of the specific enterprise. Fig. 7 - Fig. 9 graphically present processes of material (stone) processing. The axis $\mathrm{X}$ presents a number of days with the maximum capacity of the machine, axis Y presents a number of pieces of the processed material. Curves with different colours present: blue curve presents number of pieces of the processed material, red curve presents the number of pieces of wastes from the processed material, green curve presents number of pieces of the processed material by the simulation experiment, grey curve presents number of pieces of waste from the simulation experiment of the processed material. 


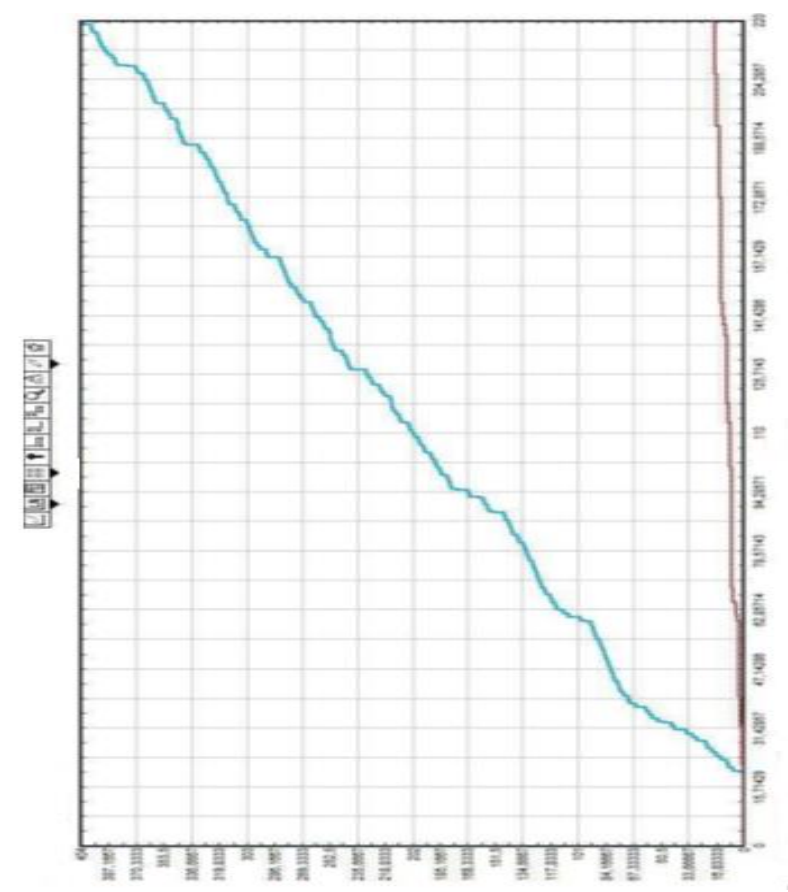

Fig. 7. Graph of processing of the polishing material. Source: Authors

Fig. 7 presents that the stone cutting machine is utilized during the whole year and the material is accumulated before the next processing.

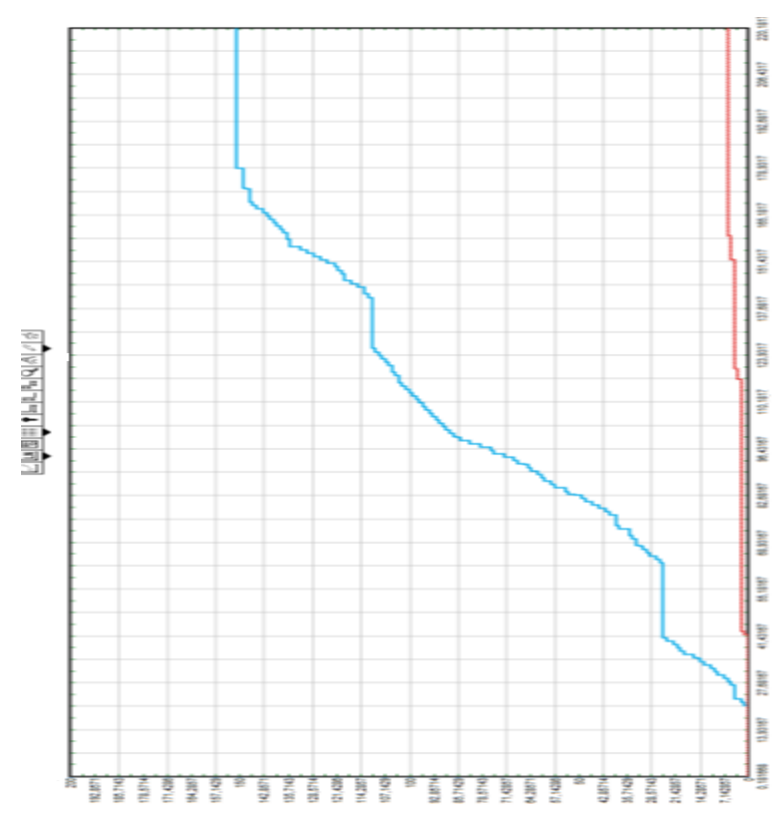

Fig. 8. Graph of flaming material processing. Source: Authors

Fig. 8 presents the process of flaming material processing. From this graph is evident that the machine for material processing by flaming is less utilized than the stone cutting machine during the year. 


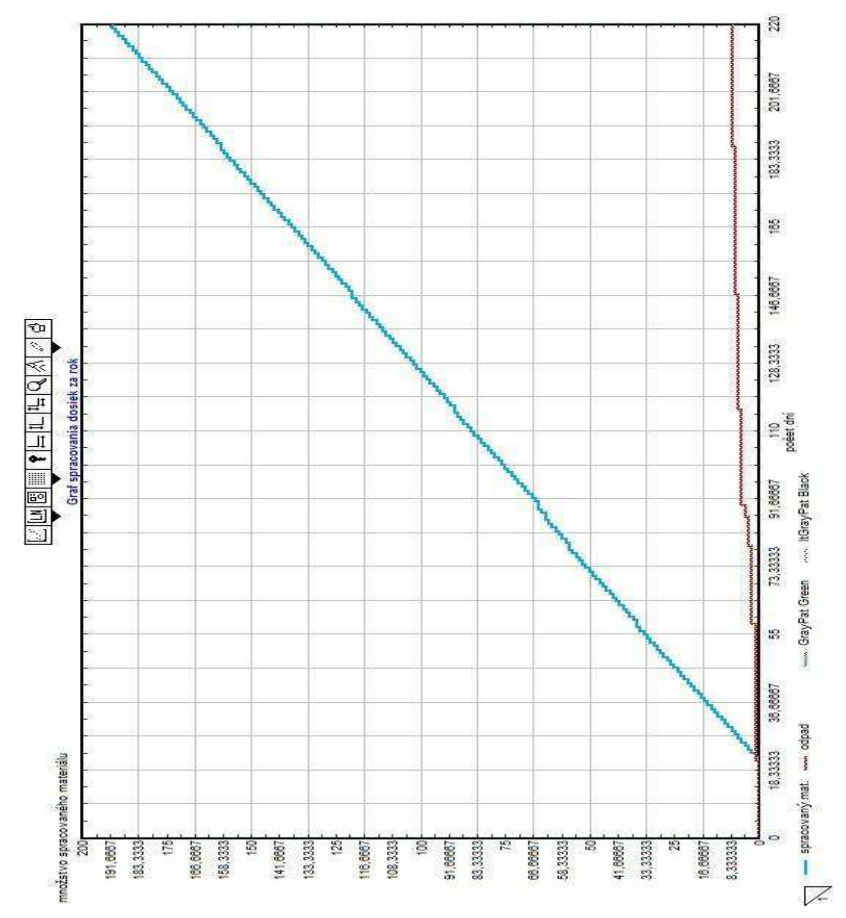

Fig. 9. Graph of the processing part - cutting of tables. Source: Authors

Fig. 9 presents that the machine is a maximum utilized full year. This shows that the material is accumulated in front of the machine and the machine does not make the material in time. In real time, if the event occurs, the enterprise will stop the input material by the time the material processing. This accumulated material is stored on the outside place, stored for storage. From the data obtained from the enterprise, this time presents many times a real onehalf year. The maximum amount of the processed material in the production process is limited by the capacity of the machine and this machine presents a bottleneck of the production process and its capacity is depleted as the first. The examined enterprise has a high demand for its material, therefore it was realized the study to eliminate this bottleneck of the production process (for an increase of the volume of the production, increase profit and satisfy the customers). After comparing the actual values with the results of the simulation experiments (Fig. 10 - Fig. 15) it is obvious that adding another three stone-cut machines and two multi-plate machines the values are identical.

Fig. 10 presents the resultant graph of the simulation experiment with the resultant graph of the simulation of processing of material polishing. After adding two stone-cut machines to the simulation experiment of material processing by polishing is graphically obviously, there is no massive accumulation of material in front of the stone-cut machine. This shows that for more effective material processing adding of two stone-cut machines is needed. 


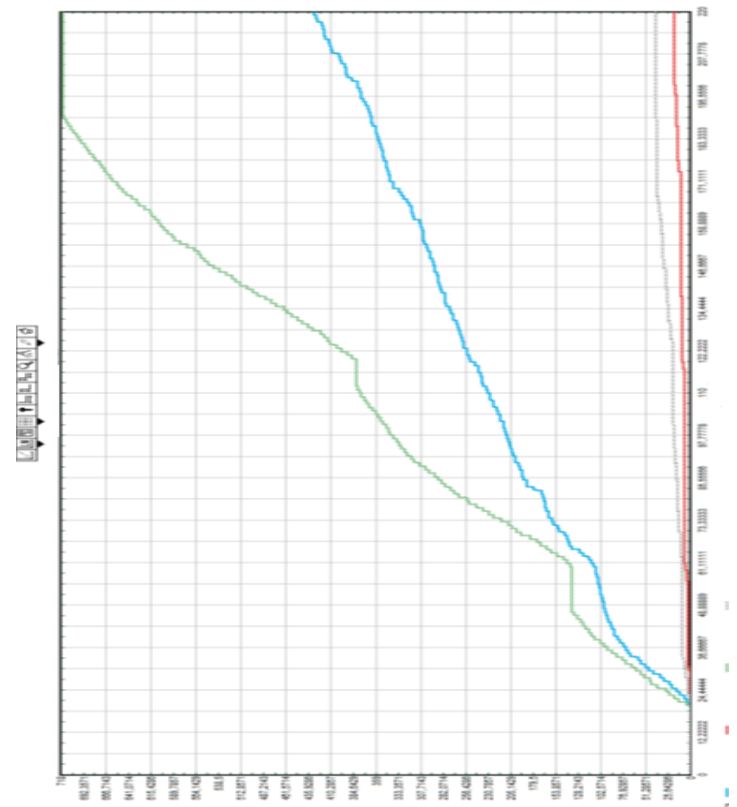

Fig. 10. Simulation experiment of the processing part - material polishing (output from the simulation model). Source: Authors

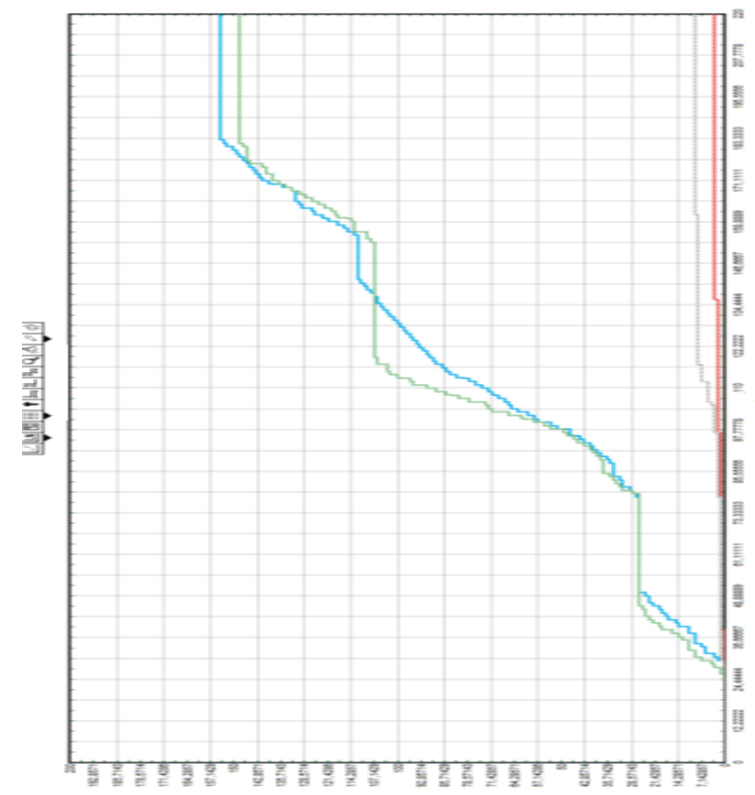

Fig. 11. Simulation experiment of the processing part - material flame (output from the simulation model). Source: Authors

During the simulation experiment, a new machine was added to the original multi-plate machine. The results of the simulation experiment are presented graphically. These values are compared with the values of the original simulation (Fig. 11). By comparing the resulting values of the graph of a simulation of flame material processing with the experiment it is evident that values differ greatly from each other. 


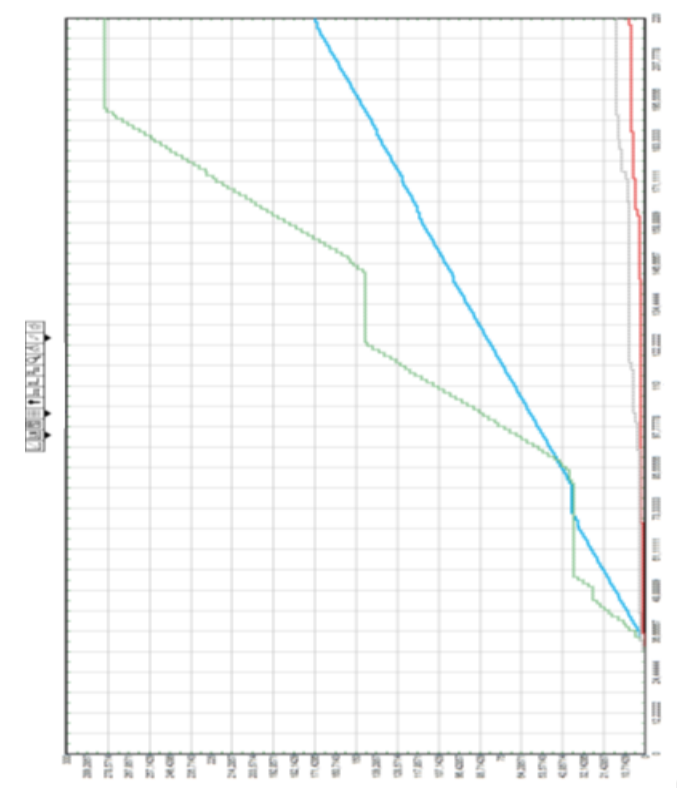

Fig. 12. Simulation experiment of the processing part - tables cutting. Source: Authors

By the help of the simulation experiment (two new mechanisms were added to the original stone-cutting machine) it is evident much material in comparison with the independent operation and also there is not accumulated material in front of the stone-cutting machine. The graph (Fig. 12) also shows the difference in the utilization of the machine when it worked independently and at the same time in cooperation with several machines.

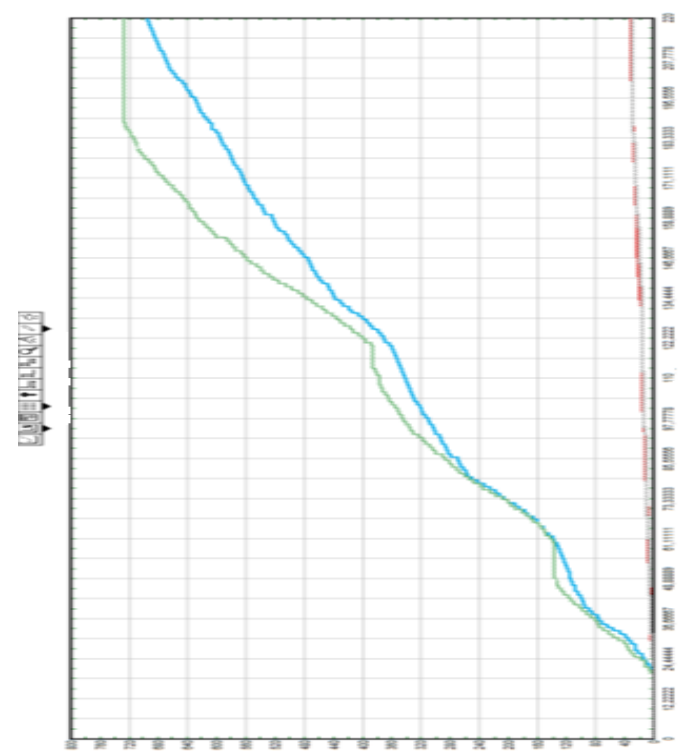

Fig. 13. Simulation experiment of the processing part - material polishing. Source: Authors

Fig. 13 presents the experiment in the case if one stone-cutting machine is an outage. The graph shows the difference between the original simulation presented by the green colour and simulation with the outage of one stone-cutting machine presented by the blue colour. 


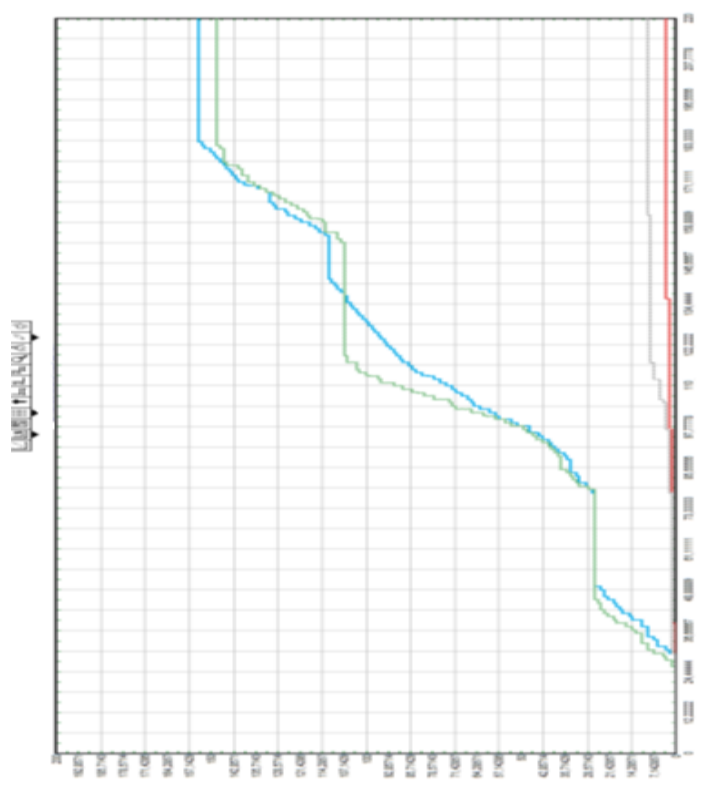

Fig. 14. Simulation experiment of the processing part - material flame. Source: Authors

This figure (Fig. 14) presents the experiment with the outage of one multi-plate machine. The graph shows that the difference between the original simulation and the experiment is not great. From this results in the fact, that the machine can work independently and will not assemble a large amount of material for processing.

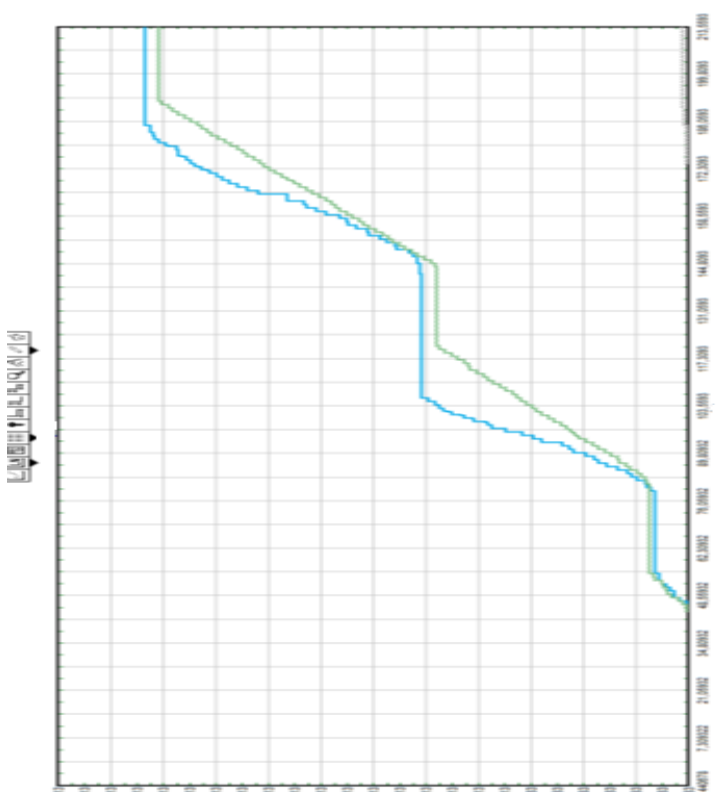

Fig. 15. Simulation experiment of the process - tables cutting. Source: Authors

Fig. 15 presents the experiment of the process of material processing by cutting. From this figure it is evident that in the case of one machine gets out of operation, it will start the material collection before the processing and the time of processing is extended. 


\section{Conclusion}

The aim of this study was to simulate the production process of stone processing in the examined enterprise. By the help of the obtained results from available documents and sources about the amount and time of material processing and also by the help of the simulation language Extend, it was created the simulation model for the simulation of the production process and its two parts. The final part of the simulation is presented by the simulation experiment, especially graphical presentation of the production process, and this shows the amount of material processed by machines. This data presents the utility of the machine (its timetable, stoppages, etc.). By the simulation, it was monitoring the accumulation of material in front of the material input, and this is the bottleneck in the production process for the examined enterprise. This problem was solved by the proposal, namely by the addition of other machines to the production process. By the simulation experiment, it is possible to simulate the production process in the case of innovations or failure of one of the machines. The simulation experiment suggests various improvements of the simulated system and it verifies the impact on the modelled system and results are reverse applied in the real system and improve its properties.

The present paper is a part of research grant projects VEGA 1/0063/16, VEGA 1/0403/18, KEGA 018TUKE-4/2016.

\section{References}

1. G. Fedorko, V. Molnár, S. Honus, H. Neradilová, R. Kampf, Int. Journal of Simul. model. 17, (2018)

2. D. Sabadka, V. Molnár, G. Fedorko, Adv. In Sc. And Technol. - Res. Journal 11 (2017)

3. S. Roth, F. J. Konig, C. Dirschl, M. Heinrich, Accurate Vehicle Simulation in Logistic and Manufacturing Planning (Simulating urban traffic scenarious, 2019)

4. S.B. Layeb, A. Jaoua, A. Jbira, Y. Makhlouf, Computer and industrial engineering 126, (2018) 